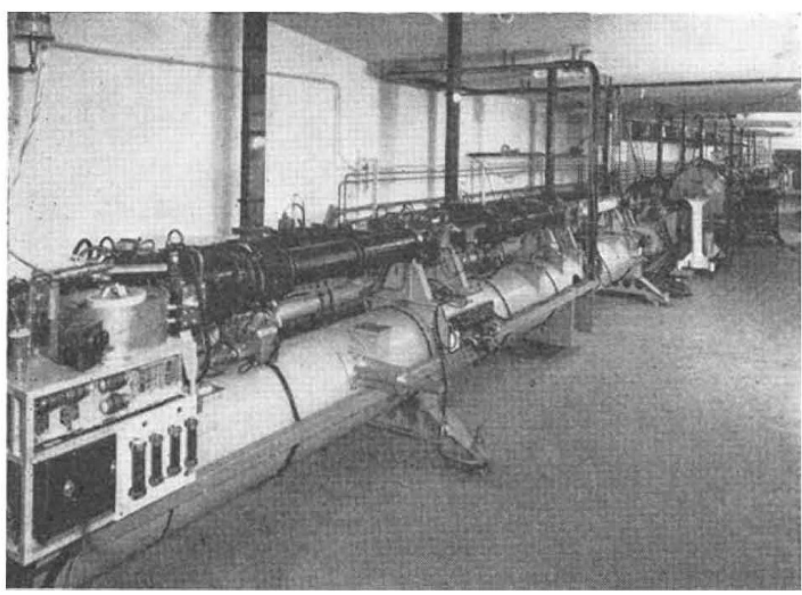

$100 \mathrm{MeV}$ electron linear accelerator at the Kelvin Laboratory of Glasgow University. The electron injector is on the left; vertical waveguides carry radio frequency power to the accelerator.

between pulses to allow the equipment to cool down. A vacuum is maintained inside the machine to avoid electrical breakdown and to prevent scattering of the electrons. Electrons leave the machine through a metal window, and are used directly in experiments, or pass through a magnetic dispersion system to select the required energy from the beam.

\section{Good News for Sheep}

For the past seven years or so veterinary surgeons and immunologists at the Wellcome Research Laboratories, Beckenham, have been busy perfecting a vaccine which simultaneously immunizes sheep against seven diseases caused by members of the genus Clostridium. The most successful answer they have come up with so far is the 'Omnivax' system-demonstrated at the Burroughs Wellcome centre at Frant on June 10.

The vaccine has a long history: Burroughs Welleome and Co. introduced the first commercially available vaccine for sheep in the $1920 \mathrm{~s}$, but it was not until 1961 that the first multi-component, seven-in-one vaccine was developed against struck, lamb dysentery, black water, tetanus, pulpy kidney, braxy and black disease. Together, these diseases have caused sheep losses costing almost $£ 1$ million. This vaccine has, however, two disadvantages. First, it is a complex of antigen and an aluminium adjuvant, the aluminium frequently causing an inflammatory reaction at the site of inoculation, leaving a persistent nodulc. Second, it is necessary to inoculate five times in two years, which is both time-consuming and costly. The new system gets over both these problems: it incorporates an oily adjuvant which does not produce an inflammatory reaction, and a single injection administered to lambs at 10-12 weeks gives protection for at least two vears.

The 'Omnivax' system has two components. The first dose of the white, milky, oily adjuvant is administered by a veterinary surgeon to ensure that the inoculation is made at precisely the right site--straight into the abdominal cavity. Other veterinary vaccines are commonly injected subcutaneously. The second component, 'Omnivax' clostridial vaccine (aqueous), is administered subcutaneously by the farmer twelve to two weeks before lambing to ensure that a high level of antibody is passed on to the suckling lamb.

Using an automatic syringe which dispenses $2 \mathrm{ml}$. doses to each sheep from a $100 \mathrm{ml}$. polythene container, veterinary surgeons have vaccinated up to 200 sheep an hour with the oily adjuvant. The cost of material to confer complete immunity on lowland ewes for a period of five years using the new system is estimated to be $4 s .6 d$. This compares with $5 s .9 d$. to $8 s .6 d$., which is the cost at present of the ten inoculations needed to confer the same immunity using conventional vaccines. On top of this there will be an additional cost to the farmer of $£ 44 s$. per hour for the services of the veterinary surgeon, as laid down by the British Veterinary Association; this cost will, however, vary according to available facilities.

Designed primarily for the breeding stock, the 'Omnivax' system gives the farmer greater flexibility in the timing of his vaccination. It will no longer be necessary to gather the flock specially for vaccination; it can be arranged to coincide with weaning, worming and other routine husbandry.

\section{Hope for Battered Babies}

The National Society for the Prevention of Cruelty to Children is in the process of setting up in Britain a special research unit to investigate the "battered baby syndrome" - a phenomenon that has been studied in the United States for the past seven years. The unit, composed of three social workers and headed by Miss Joan Court, a psychiatric social worker, will begin operation in October and expects to combine research and casework in intensive work with a small number of families, 35 to 40 . The families will probably come from within a small area so that a caseworker may be available on a 24 hour basis over a period of three to five years.

A book on battered children recently published in the United. States (The Battered Child, edit. by R. E. Helfer and C. H. Kempe, University of Chicago Press, 1968) reviews the work being done on this subject in America and estimates that between two and three thousand young children are injured each month and usually one or two are killed every day in the US from non-accidental repeated physical abuse by parents or guardians. Taking these estimates as a guide, there are probably three to four hundred cases in Great Britain each year, though most of these probably go unrecognized and unreported.

Dr Helfer, a pacdiatrician and one of the editors of the book, stresses that the people who inflict these injuries on their children come from all walks of life, and there are indications that the situation is found in all countries. From the psychiatric studies made so far, it would seem that these are not cases of premeditated torture or death, but only occur when a child is unable to satisfy an adult's emotional needs. These adults, instead of providing for the emotional needs of their child, expect the child to satisfy their needs and therefore anticipate that the child will be able to do things it is as yet physically unable to do; this causes them, in moments of stress, to attack the child. Studies mado seem to indicate that adults who batter their children were battered themselves as children, indicating a continuing and self-perpetuating syndrome. 